\title{
Optical characterization of the PALM-3000 3388-actuator deformable mirror
}

Jennifer Roberts, Antonin H. Bouchez, Rick S. Burruss, Richard G. Dekany, Stephen R. Guiwits, et al.

Jennifer Roberts, Antonin H. Bouchez, Rick S. Burruss, Richard G. Dekany, Stephen R. Guiwits, Mitchell Troy, "Optical characterization of the PALM-3000 3388-actuator deformable mirror," Proc. SPIE 7736, Adaptive Optics Systems II, 77362E (28 July 2010); doi: 10.1117/12.857815

SPIE Event: SPIE Astronomical Telescopes + Instrumentation, 2010, San Diego, California, United States 


\title{
Optical Characterization of the PALM-3000 3388-Actuator Deformable Mirror
}

\author{
Jennifer Roberts* ${ }^{1}$, Antonin H. Bouchez ${ }^{2}$, Rick S. Burruss ${ }^{1}$, Richard G. Dekany ${ }^{2}$, Stephen R. \\ Guiwits ${ }^{1}$, Mitchell Troy ${ }^{1}$ \\ ${ }^{1}$ Jet Propulsion Laboratory, California Institute of Technology, Pasadena, CA, 91109 \\ ${ }^{2}$ Caltech Optical Observatories, California Institute of Technology, Pasadena, CA, 91125
}

\begin{abstract}
We describe the lab characterization of the new 3,388-actuator deformable mirror (DM3388) produced by Xinetics, Inc. for the PALM-3000 adaptive optics (AO) system ${ }^{1}$ under development by Jet Propulsion Laboratory and Caltech Optical Observatories. This square grid 66-by-66 actuator mirror has the largest number of actuators of any deformable mirror currently available and will enable high-contrast imaging for direct exoplanet imaging science at the Palomar 200" diameter Hale Telescope. We present optical measurements of the powered and unpowered mirror surface, influence functions, linearity of the actuators, and creep of the actuators. We also quantify the effect of changes in humidity.
\end{abstract}

Keywords: deformable mirror, Xinetics, Palomar, adaptive optics, PALM-3000

\section{INTRODUCTION}

The DM3388 is the heart of the PALM-3000 AO system. It will give the system the ability to control wavefront with $8 \mathrm{~cm}$ sampling on the sky, for the highest spatial frequency correction by any AO system currently in operation or in development. The primary science goal of the PALM-3000 upgrade is to provide high-contrast imaging and spectroscopy, when combined with the P1640 coronagraphic integral field spectrograph. PALM-3000 will also support general use near-infrared and visible imaging and spectroscopy, using natural guide stars (NGS). Performance of $105 \mathrm{~nm}$ residual RMS wavefront error (WFE), better than $10^{-6}$ contrasts in J-band, $90 \% \mathrm{~K}$-band Strehl ratio and $60 \%$ I-band Strehl is expected using NGS brighter than $V=6.5$, with usable correction with lower performance to $V=17$. In addition to the 3,388 actuator DM, PALM-3000 will also include a Xinetics 349-actuator DM (DM349) which will control up to $4 \mu \mathrm{m}$ peak-to-valley of low-spatial frequency aberrations (up to 8 cycles across the pupil).

\subsection{Requirements}

The initial optical specifications for the 3,388 actuator DM are given in Table 1. The surface figure requirements were not met, but testing has determined that the DM surface figure will provide acceptable performance for the PALM-3000 system. The uncorrected surface figure value is addressed in more detail in Section 5. Since DM349 will correct lowspatial frequency aberrations, the high-spatial frequency content of the residual figure of DM3388 is of primary interest.

Table 1 Optical Requirements

\begin{tabular}{|l|l|l|}
\hline Characteristic & Required Value & Achieved Value \\
\hline Uncorrected Surface Figure & $\lambda / 30 \mathrm{RMS}$ & $\lambda / 5 \mathrm{RMS}$ \\
\hline Corrected Surface Figure & $\begin{array}{l}<5 \mathrm{~nm} \text { RMS (Goal) } \\
<10 \mathrm{~nm} \text { RMS (Requirement) }\end{array}$ & $11 \mathrm{~nm}$ RMS \\
\hline Total Stroke & $>1 \mu \mathrm{m}$ & $1.06 \mu \mathrm{m}$ \\
\hline Interactuator Stroke & $>0.25 \mu \mathrm{m}$ & $0.5 \mu \mathrm{m}$ \\
\hline
\end{tabular}

*jennifer.roberts@jpl.nasa.gov; 818-354-1304; http://www.oir.caltech.edu/twiki_oir/bin/view/Palomar/Palm3000/WebHome

Adaptive Optics Systems II, edited by Brent L. Ellerbroek, Michael Hart, Norbert Hubin, Peter L. Wizinowich, Proc. of SPIE Vol. 7736, 77362E - C 2010 SPIE · CCC code: 0277-786X/10/\$18 · doi: 10.1117/12.857815 


\subsection{Experimental Data}

The DM3388 was tested in the adaptive optics lab at Caltech Optical Observatories. All data were taken with a 6" aperture Zygo interferometer with a $1 \mathrm{kx} 1 \mathrm{k}$ pixel detector, giving a sampling of $\sim 15$ pixels per actuator. The high pixel density was required to accurately measure the high spatial frequencies present across the aperture. There was a single $\lambda / 20$ fold flat between the DM3388 and the interferometer. All measurements are microns of surface error.

Because space constraints on the test bench required a path length of a couple of meters, there was some noise in the measurements due to air turbulence. Several measurements were averaged to reduce the effect, but it produced mostly low-spatial frequency disturbances, so it did not affect the primary goals of the experiment.

\section{MIRROR DESCRIPTION}

\subsection{Structure}

The mirror actuators are made of lead magnesium niobate (PMN) which is electrostrictive. The structure of the mirror is constructed of $6 \times 6$ modules of this material, each comprised of $11 \times 11$ actuators. The actuators are spaced $1.8 \mathrm{~mm}$ centerto-center with $0.15 \mathrm{~mm}$ gaps between the actuators. A $0.2 \mathrm{~mm}$ thick glass facesheet is attached to the actuators. The mirror has 3388 active actuators within the clear aperture of $117 \mathrm{~mm}$. The AO relay produces a $114 \mathrm{~mm}$ pupil which uses 64 actuators across the pupil. The entire DM package is $23 \times 23 \times 13.2 \mathrm{~cm}$.

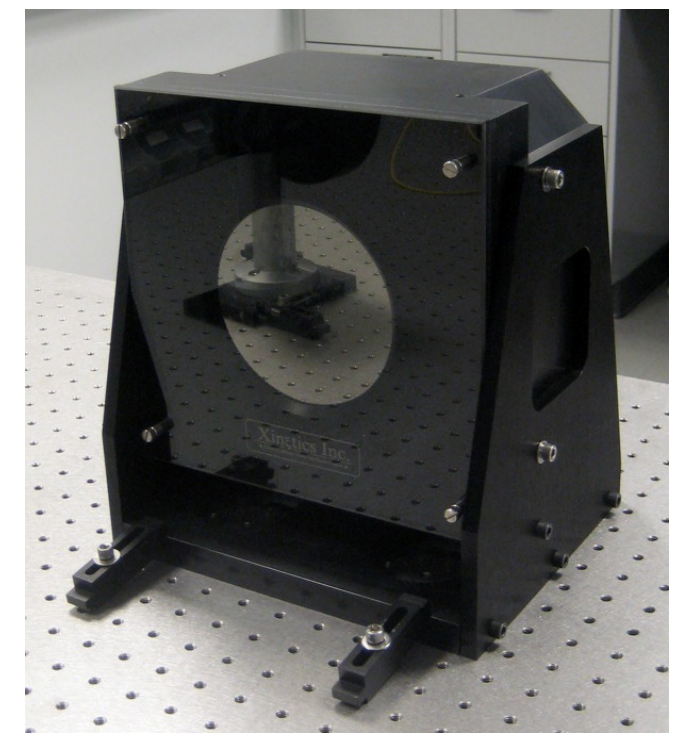

Figure 1. Xinetics 3388-actuator DM during initial testing at Caltech Optical Observatories

\subsection{Operation}

An initial voltage is applied to all the actuators to bias them to the center of their range. The actuators contract to pull the facesheet down to this bias voltage. The nominal bias voltage is $50 \mathrm{~V}$ (out of a possible $100 \mathrm{~V}$ total), but we determined experimentally that a $40 \mathrm{~V}$ bias signal better suits the needs of the project (section 3.1). The full voltage range of the mirror is limited by the driver electronics at $\pm 30 \mathrm{~V}$ from bias.

\subsection{Actuators}

Each actuator has a gaussian-shaped influence on the glass facesheet. The actuators in this mirror are square rather than round, so the shape of the influence function also has a square form. The total stroke for one actuator is $1.1 \mu \mathrm{m} \pm 0.1 \mu \mathrm{m}$, where the deviation is due to gain variations that are specific to the manufacture of the actuator material (Figure 2). Xinetics selected 11 actuators across a module in order to minimize gain variations across a module. 


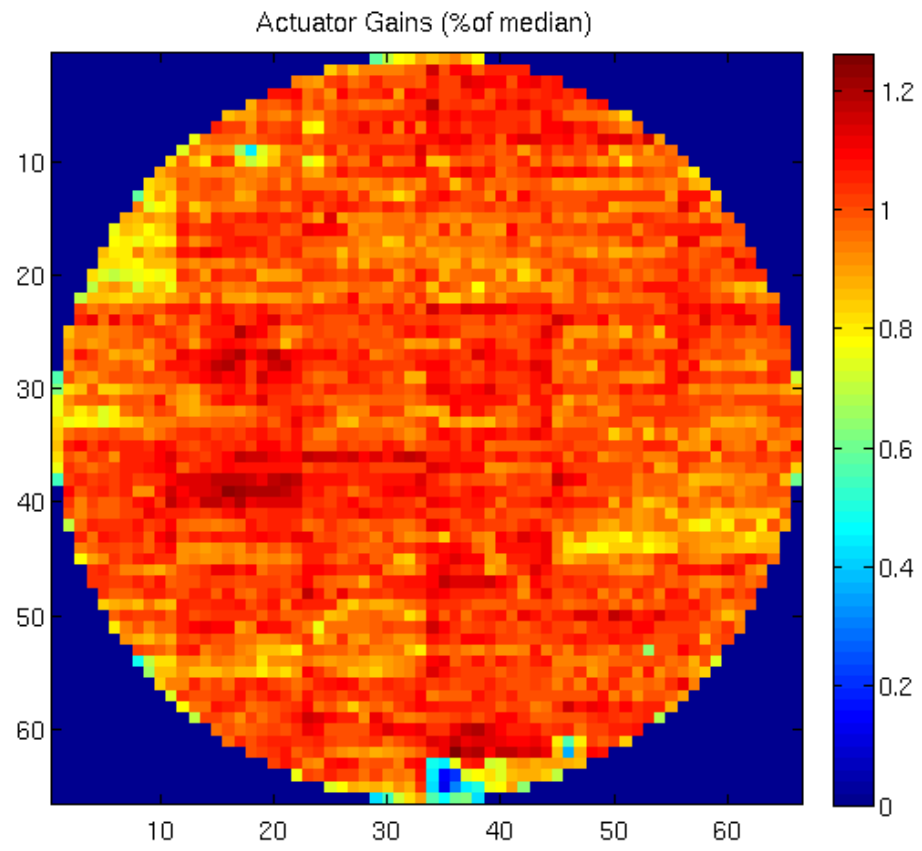

Figure 2.Actuator gain variation (see Section 5.1 for more detail)

The profiles of the influence functions overlap at approximately the FWHM of the Gaussian so that it is possible to piston the entire mirror (Figure 3). This also reduces the high-spatial frequency structure induced when controlling the entire mirror.
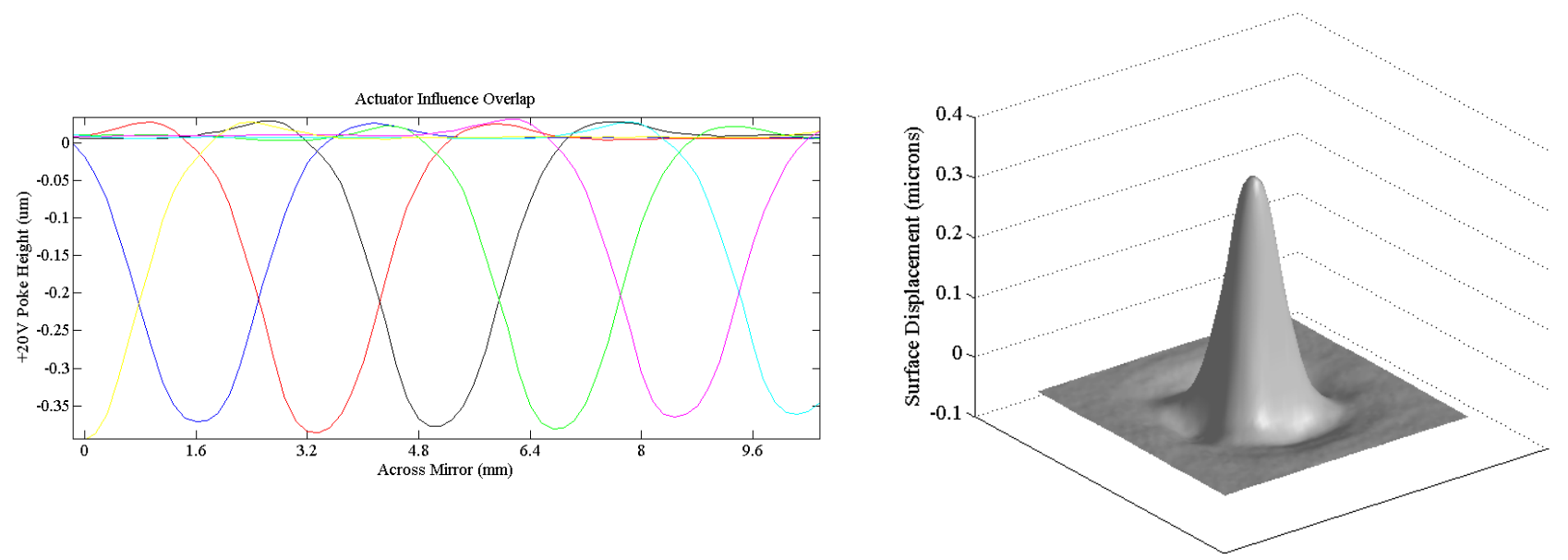

Figure 3.Measured actuator pokes $(20 \mathrm{~V}$ out of $\pm 30 \mathrm{~V})$. Adjacent influence function overlap (left); Shape of single poke (right)

\section{ACTUATOR PROPERTIES}

\subsection{Linearity}

Several properties of the actuators are critical to their use in PALM-3000. Knowing the linearity of the gain over the full voltage range is required for the servo control to work properly to meet our rigorous performance requriements. Regardless of bias voltage, the actuators are very linear over a $\pm 0.1 \mu \mathrm{m}$ range. The larger the bias, the more the material 
contracts initially and the less range there is available for individual actuator stroke. So, at larger bias signals, the total stroke of the actuators is smaller, but the gain is more linear over the available voltage range. For a range of bias values from $35 \mathrm{~V}$ to $65 \mathrm{~V}$, all actuators were measured at $3 \mathrm{~V}$ intervals over the full voltage range, $-30 \mathrm{~V}$ to $+30 \mathrm{~V}$. Based on the measured curves (Figure 4), we determined that a $40 \mathrm{~V}$ bias signal represented the best tradeoff. The nonlinearity over the voltage range at $40 \mathrm{~V}$ bias will need to be accounted for in the servo control software. The manufacturing defect discussed in section 5.1, is also improved at lower bias voltages because the mirror was polished in an unpowered state.
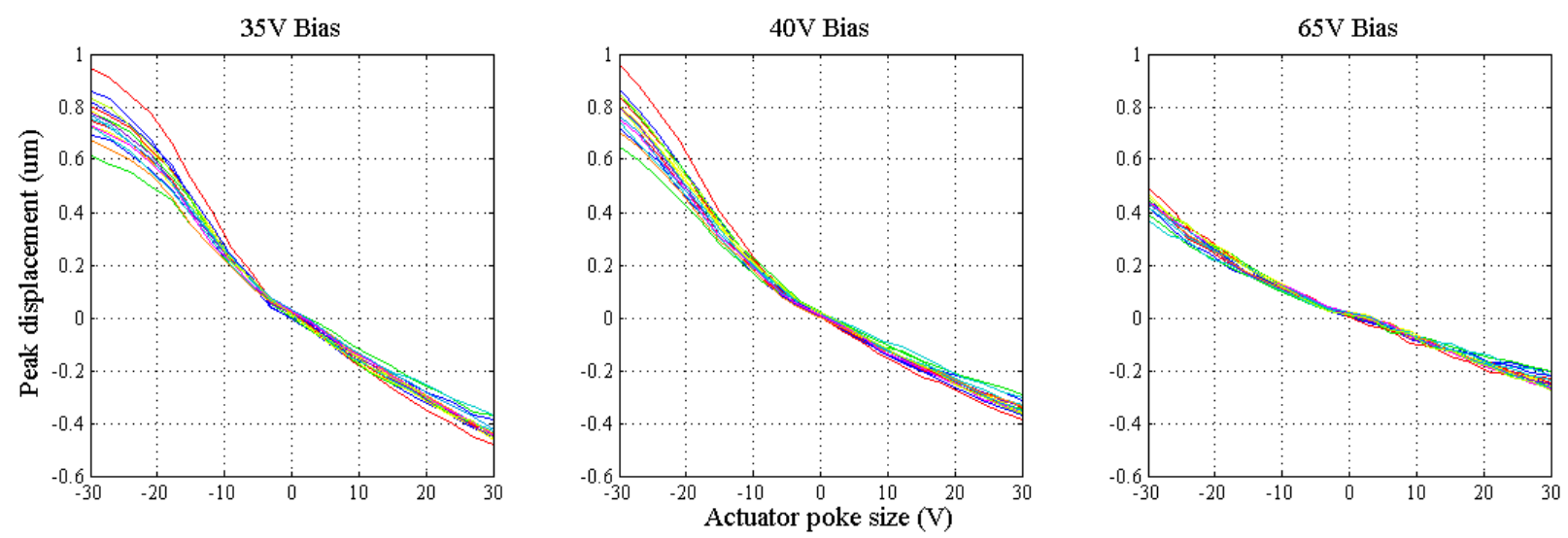

Figure 4.Affect of bias voltage on actuator linearity, as represented by surface displacement, for a representative sample of actuators.

\subsection{Hysteresis and Creep}

Other important properties of the actuators are hysteresis and creep. Hysteresis manifests itself as the inability to return to the same actuator height given the same voltage. Creep is a change in actuator position over time with no change in commanded voltage. In closed loop, neither of these effects poses a problem for the operation of the AO system. For static operations, such as applying the best flat commands during setup and alignment, both properties will have an effect. The effect of creep is proportional to the size the voltage change and the length of time since the change was applied. Moving the actuators to bias at first power up is the equivalent of $40 \mathrm{~V}$ pokes. While the effect is significant (Figure 5), it is easily mitigated by putting the mirror at bias voltage or best flat voltages at least an hour before use.
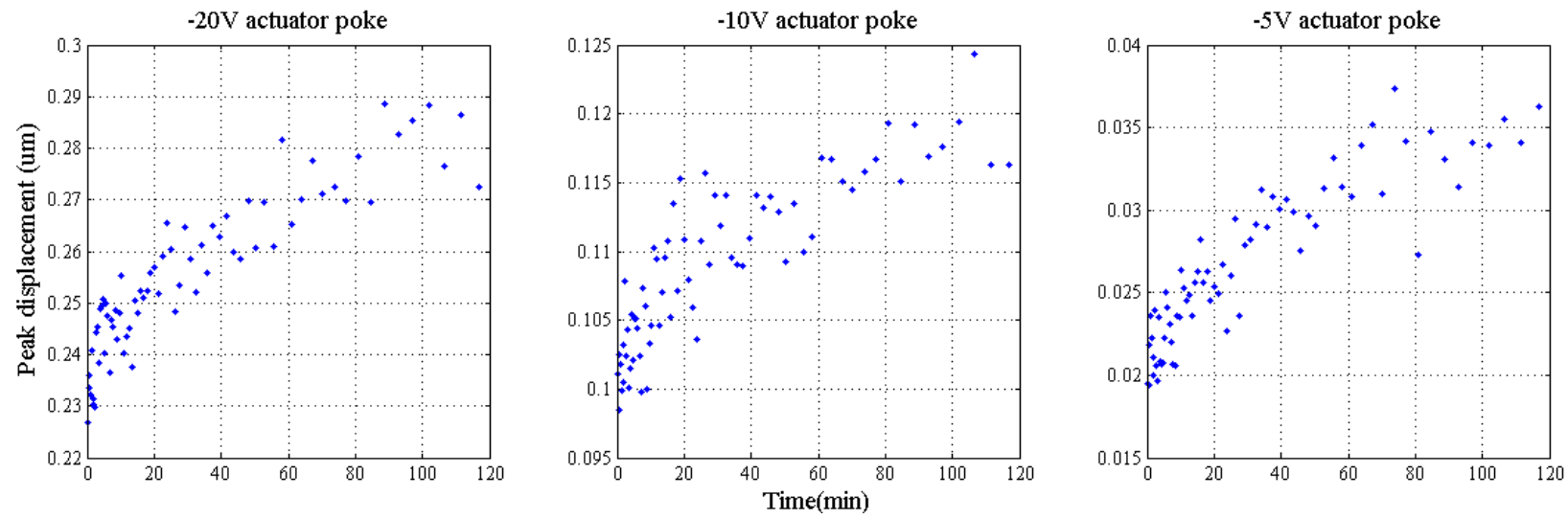

Figure 5. Size of creep effect by total size of poke (33\%, $17 \%$ and $8 \%$ of the total range, respectively).

With the serial interface to the electronics that was available during testing, the mirror voltages had to be set to zero before each new set of commands was sent. Using this capability, the repeatability of actuator commands was measured to be $\pm 16 \mathrm{~nm}$. But because of the control limitation, it was not possible to properly measure the hysteresis of the actuators. This will be checked with the normal high speed interface to the electronics once it is available. 


\section{MIRROR CONTROL}

\subsection{Best flat}

The mirror was controlled using a simple, measured, least-squares reconstructor. All actuators were measured using patterns of well separated actuators. To reduce effects of noise in the measured influence functions, an average influence function was created from individual measurements. This average measurement, the measured gains and the measured location of each actuator were used to create the reconstructor between Zygo measurements and DM actuator motion.

Using this method, the best controlled flat gives $11 \mathrm{~nm}$ RMS surface error (Figure 6). This measurement was made with only the DM3388 providing the correction, so the total stroke used is $12 \%$ RMS of the available actuator stroke, with several individual actuators using up to $40 \%$ of their stroke. If we assume that spatial frequencies of 8 cycles or less across the pupil will be corrected by the low-order DM, then this surface figure is achieved using less than 5\% RMS of the available actuator stroke with a few individual actuators using up to $25 \%$ of their stroke.

The large grid (6x6) structure is due to variations from module to module. The material is sensitive to humidity and temperature, which produces a slight cupping affect from the edge to the center of each module. This effect produces errors on the order of $100-200 \mathrm{~nm} \mathrm{P}-\mathrm{V}$ and is well within the range correctable by the low-order DM. The larger fine structure in the flattened surface (red and blue areas) is due to the actuators being unable to control manufacturing defects (Section 5.1) between the actuators. The smaller fine structure is inherent to the manufacture of the actuator modules.
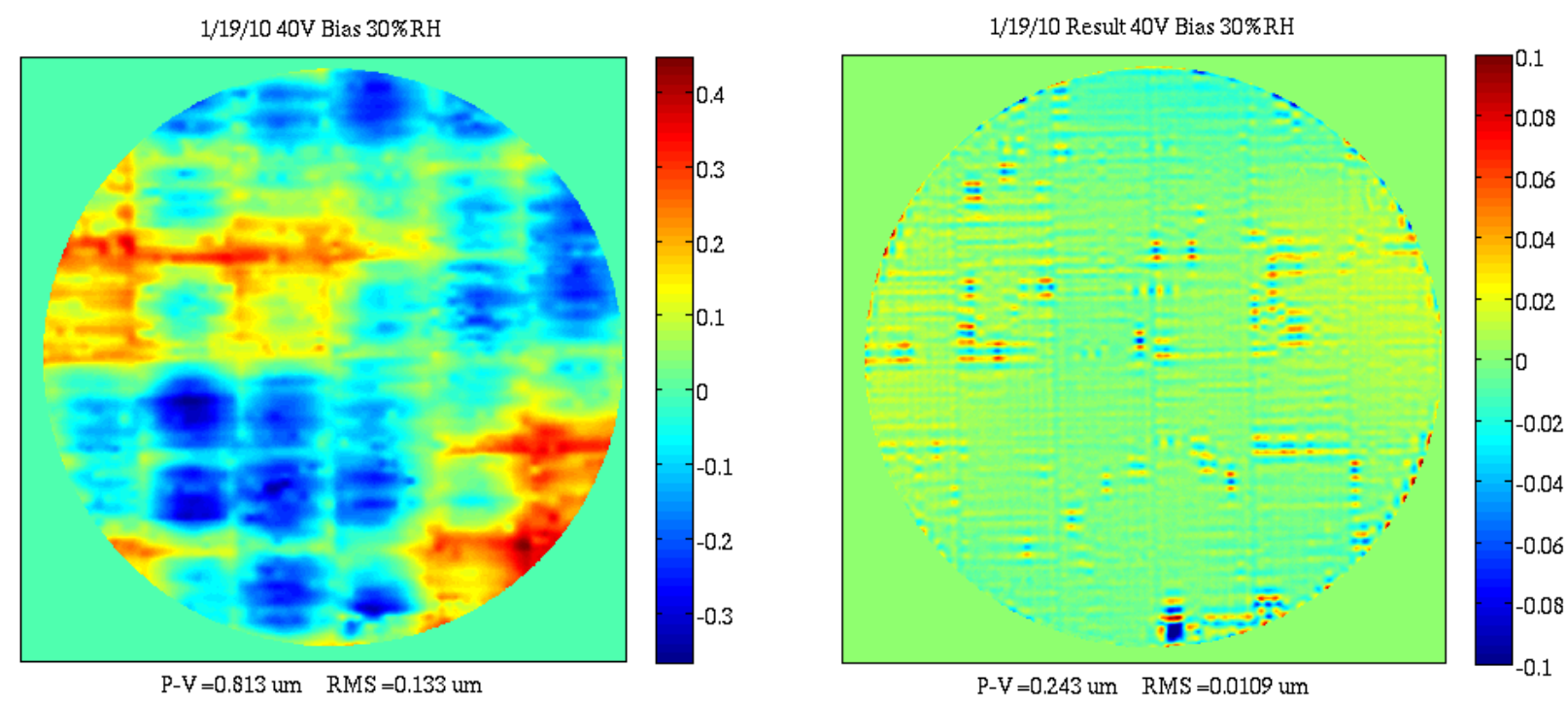

Figure 6.Mirror figure at bias voltage, all actuators at midrange (left); Best controlled mirror figure (right). 


\subsection{Stroke measurement}

Individual actuator pokes can invoke interactuator stroke limits which are set to a difference of $30 \mathrm{~V}$ volts, or half the range, between adjacent actuators. To test the full stroke of the actuators, the mirror was controlled to a smooth global shape, in this case focus, which maximized the use of the available voltage (Figure 7). The peak-to-valley is the maximum stroke of the mirror, $1.06 \mu \mathrm{m}$.
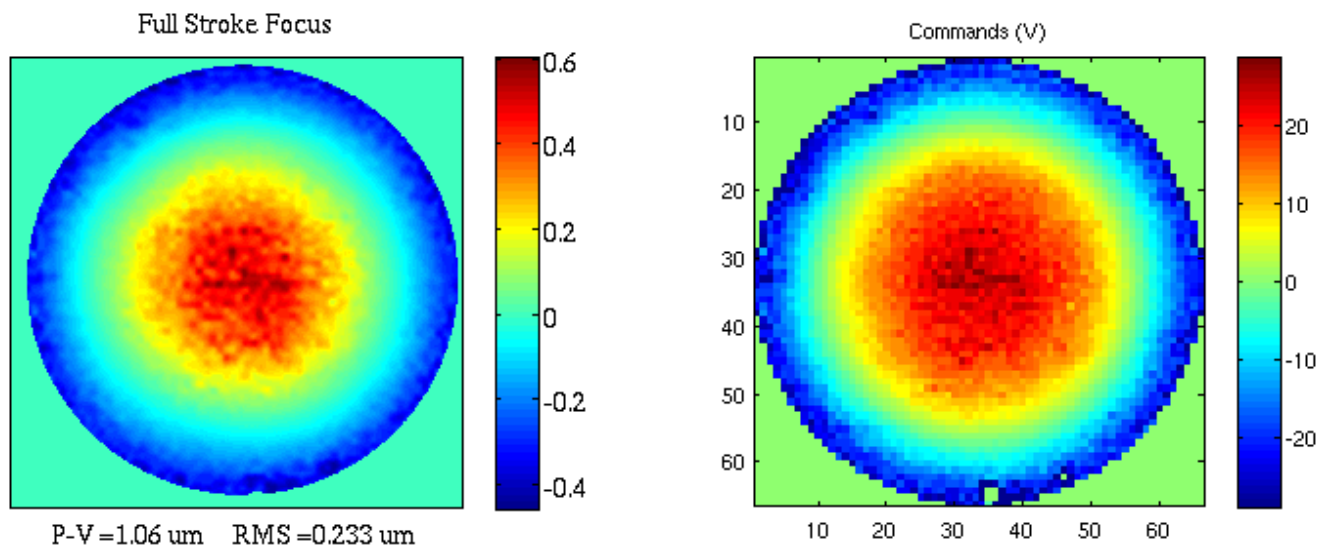

Figure 7. Full stroke measured using a global slope across mirror (focus) - Total 1.06um P-V

\section{HUMIDITY}

\subsection{Manufacturing defect}

Although the DM3388 represents a significant prototyping achievement for Xinetics, there were imperfections created during the manufacturing process which affect the surface shape of the mirror.

Because the actuators are very closely spaced and are made of an electrically active material, the entire grid of actuators is dipped in a conformal coating before the facesheet is attached. During initial polish of the completed mirror, polishing slurry got into the area between the actuators. This caused either the conformal coating or the adhesive used to attach the facesheet to absorb moisture and wick between the actuators. When the mirror was coated in vacuum, the material dried out and was fixed in place between actuators. Thus, as the material absorbs or releases moisture, it expands and contracts. The actuators are firmly attached to the facesheet, so as the material expands or contracts, the spacing between actuators varies accordingly and causes puckers in the facesheet (Figure 8). For the majority of affected actuators, the stroke of the actuator is not affected. There is an area on the bottom edge of the mirror which is more severely affected and 18 of those actuators do have reduction to less than half the nominal stroke (Figure 2). 8 of the actuators with reduced stroke are inside the clear aperture that will be used in the PALM-3000 system.
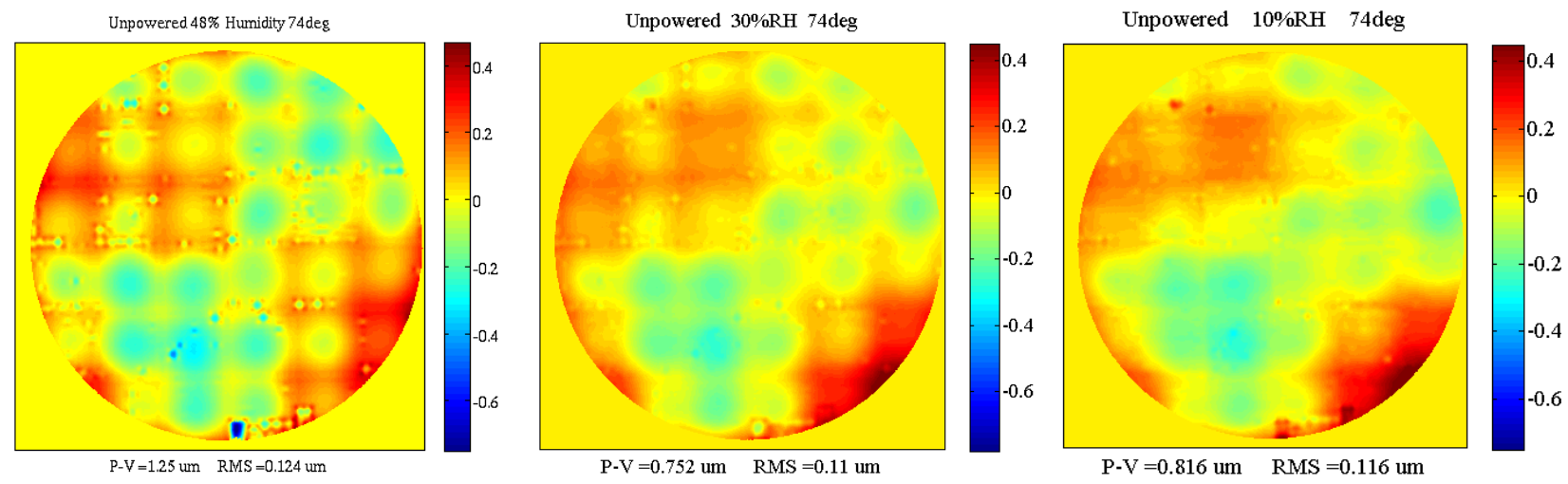

Figure 8.Unpowered mirror figure at a range of humidities 
The complete solution to the problem would be to remove the facesheet and attempt to clean between the actuators. This would likely damage the facesheet and require use of the only spare facesheet. There is also a significant risk of permanently damaging the actuators. Due to this risk, time and financial constraints, this solution will not be pursued in the near future. Alternatively, we elected to repolish the mirror at a controlled humidity of $30 \% \mathrm{RH}$, minimizing the figure error, and adopting a strategy of humidity control.

\subsection{Humidity Control}

To keep the actuator material at this specific humidity, it was necessary to seal the mirror case. This included gaskets around the front and back plates of the mirror case, as well as using a low outgassing RTV material to seal each of the 99 connectors on the back plate. Instead of a normal desiccant, which would produce too low a humidity, we are using a material that absorbs and releases moisture in order to keep a specific humidity, in this case $33 \% \mathrm{RH}^{2}$. The sealing and desiccant material have proved to be effective in keeping the humidity inside the DM to $32-34 \%$ in a lab environment where the outside humidity has ranged from $30-60 \%$ and the temperatures have ranged from $65-85^{\circ} \mathrm{F}$.

\subsection{Results}

Once the humidity in the DM had stabilized (more than a week at $33 \% \mathrm{RH}$ ), measurements were taken each day using the best flat commands from all previous days as well as best flat commands calculated for that day. A comparison of the surface error from each set of commands showed variations in the low spatial frequencies on the order of $\lambda / 10$ RMS. But the variability in the high spatial frequencies (greater than 8 cycles across the pupil) was only $3 \mathrm{~nm}$ RMS surface error (Figure 9), within the performance tolerance of PALM-3000's most demanding science requirement for better than $10^{6}$ coronagraphic exoplanet imaging contrast.

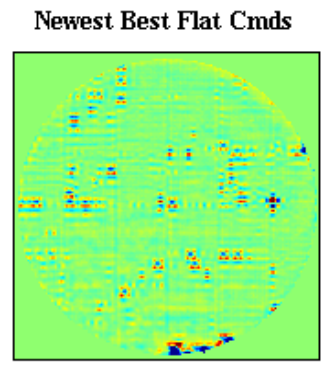

RMS $=0.0214$ um

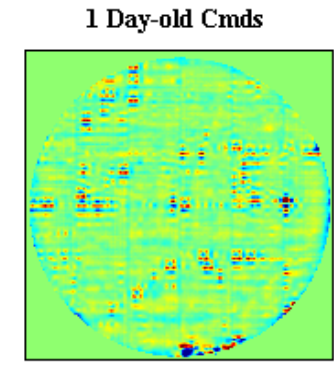

RMS $=0.025$ um

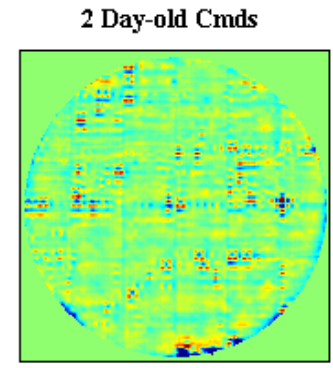

RMS $=0.0261$ um

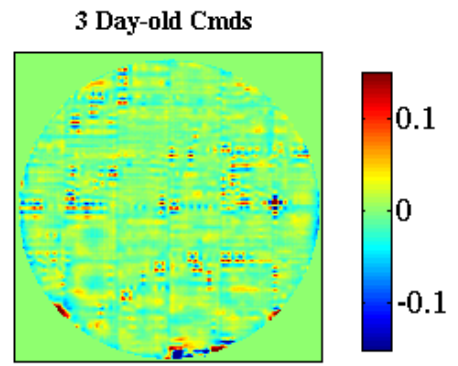

RMS $=0.0262$ um

Figure 9.The high spatial frequency content (greater than 8 cycles across the pupil) of the mirror surface. All data were taken on the same day using the current best flat commands and the best flat commands from previous days.

\section{CONCLUSION}

Xinetics, Inc. has produced a new 3,388-actuator DM that will work well in the PALM-3000 system. It provides the required spatial wavefront correction, with enough stroke to meet the key science performance requirements on the sky. Despite an unexpected sensitivity to humidity tracable to the prototypical nature of the manufacturing process and now correctable by the manufacturer, our testing at Caltech Optical Observatories has demonstrated sufficient humidity control to enable PALM-3000 science.

\section{ACKNOWLEDGEMENTS}

This research was carried out in part at the Jet Propulsion Laboratory, California Institute of Technology, under a contract with the National Aeronautics and Space Administration. The DM was funded by the NASA SBIR program.

Reference herein to any specific commercial product, process, or service by trade name, trademark, manufacturer, or otherwise, does not constitute or imply its endorsement by the United States Government or the Jet Propulsion Laboratory, California Institute of Technology. 


\section{REFERENCES}

[1] A.H. Bouchez et al., "Status of the PALM-3000 high-order adaptive optics system", Proc. SPIE Optics and Photonics 2009 (2009).

[2] Humidipak, http://www.humidipak.com/

Proc. of SPIE Vol. 7736 77362E-8

Downloaded From: https://www.spiedigitallibrary.org/conference-proceedings-of-spie on 7/13/2018 Terms of Use: https://www.spiedigitallibrary.org/terms-of-use 